
\title{
28 Research Square \\ LncRNA-mRNA expression pattern in invasive pituitary adenomas: A microarray analysis
}

\section{Chao Peng}

Guangdong Provincial People's Hospital

\section{Shuaikai Wang}

Shenzhen Luohu People's Hospital

Jinxiu Yu

the Second Affiliated Hospital of Guangzhou Medical University

\section{Xiaoyi Deng}

the Second Affiliated Hospital of Guangzhou Medical University

\section{Zhishan Chen}

the Second Affiliated Hospital of Guangzhou Medical University

Huiyu Ye

the Second Affiliated Hospital of Guangzhou Medical University

Hongru Yao

Guangzhou Medical University

Hanjia Cai

Guangzhou Medical University

Yanli Li ( $\sim$ liyanli@gzhmu.edu.cn )

the Second Affiliated Hospital of Guangzhou Medical University

Yong Yuan

the Second Affiliated Hospital of Kunming Medical University

\section{Research Article}

Keywords: IncRNAs, biomarker, invasiveness, pituitary adenoma

Posted Date: January 18th, 2022

DOI: https://doi.org/10.21203/rs.3.rs-1115969/v2

License: (c) (1) This work is licensed under a Creative Commons Attribution 4.0 International License.

Read Full License 


\section{Abstract}

Backgrounds

Long non-coding RNAs (IncRNAs) play important roles in tumorigenesis and progression of various cancer types; however, their roles in the development of invasive pituitary adenomas (PAs) remain to be investigated.

Methods

IncRNA microarray was performed in three invasive and three noninvasive PAs. Gene Ontology (GO) enrichment and Kyoto Encyclopedia of Genes and Genomes (KEGG) analysis were performed, and coexpression networks between IncRNA and mRNA were constructed. Furthermore, three differentially expressed IncRNAs were selected for validation by real-time quantitative reverse transcription polymerase chain reaction (qRT-PCR) in PA samples. The diagnostic values of these three IncRNAs were further evaluated by receiver operating characteristic (ROC) analysis.

Results

A total of 8872 IncRNAs were identified in invasive and paired noninvasive PAs using IncRNA microarray. Among these, the differentially expressed IncRNAs included 81 that were upregulated and 165 that were downregulated. GO enrichment and KEGG pathway analysis showed that these differentially expressed IncRNAs were associated with post-translational modifications of proteins. Furthermore, we performed target gene prediction and coexpression analysis. The interrelationships between the IncRNAs and mRNAs with significant differential expression were identified. Additionally, three differentially expressed IncRNAs were selected for validation in 41 PA samples by qRT-PCR. The expression levels of FAM182B, LOC105371531, and LOC105375785 in the invasive PAs were significantly $(P<0.05)$ lower than in the noninvasive PAs, and these results were consistent with the microarray data. ROC analysis suggested that FAM182B and LOC105375785 expression levels could be used to distinguish invasive PAs from noninvasive PAs.

Conclusion

Our findings demonstrated the IncRNAs expression patterns in invasive PAs. Thus, FAM182B and LOC105375785 may be involved in the invasiveness of PAs and serve as new candidate biomarkers for the diagnosis of invasive PAs.

\section{Background}

Pituitary adenomas (PAs), one of the most common intracranial tumors, account for $10-20 \%$ of intracranial tumors [1]. According to the tumor biological characteristics, PAs can be divided into noninvasive pituitary adenoma (NIPA), invasive pituitary adenoma (IPA), and pituitary adenocarcinoma [2]. IPAs, characterized by high proliferative and invasive activities, tend to invade vital surrounding 
structures, such as the cavernous sinus, sphenoid bone, and cranial nerves [3]. The aggressive behavior of IPAs leads to a lower cure rate of complete removal by surgical resection and a higher incidence of recurrence. Therefore, the identification of novel biomarkers for early diagnosis that reflect the clinicopathological behaviors of IPAs is important. Additionally, exploration of the molecular mechanisms involved in the invasiveness of PAs is urgently needed.

IncRNAs, with lengths $>200$ nucleotides, are involved in various processes of gene regulation, such as nuclear and cytoplasmic trafficking, chromosome dosage compensation, mRNA splicing, and translation [4]. With the development of high-throughput sequencing technologies, accumulating evidence indicates that the expression of IncRNA is associated with various tumors [5, 6]. Furthermore, IncRNAs have been increasingly identified as novel diagnostic and prognostic markers of various tumors [7, 8]; however, the roles of IncRNAs in PAs remain to be further investigated.

Xue et al.[9] have identified differentially expressed IncRNAs in PAs and revealed the key IncRNAs associated with the progression of PAs. Moreover, Guo et al.[10] showed that critical IncRNAs were associated with the recurrence of non-functioning PAs, while Zhu et al. [11] demonstrated that increased expression levels of the IncRNA maternally expressed 8 promote bone destruction in bone-invasive PAs by regulating miR-454-3p/tumor necrosis factor (TNF)-a. Exploring the expression patterns of IncRNAs in IPAs might confirm the existence of novel potential biomarkers for the diagnosis of IPAs.

This study aimed to investigate the expression pattern of IncRNAs in both invasive and noninvasive PAs. We performed microarray experiments to reveal the expression profiles of IncRNAs in three IPAs and three NIPAs. Then, we identified the expressed IncRNAs in IPAs and constructed the IncRNAs-mRNAs networks. Finally, among the differentially expressed IncRNAs, three IncRNAs were selectively examined by real-time quantitative reverse transcription polymerase chain reaction (qRT-PCR) in a large sample size for further validation, and they were applied for the diagnosis of IPAs. These findings could provide a novel insight into understanding the mechanisms of the invasive behaviors of PAs. Perhaps, IncRNAs may even be novel biomarkers for the diagnosis of IPAs.

\section{Materials And Methods}

\section{Patients and samples}

Tumor specimens were obtained from patients with PAs, who underwent transsphenoidal surgery at the Department of Neurosurgery of the Guangdong Provincial People's Hospital (Guangzhou, China) between January 2020 and June 2021. The diagnosis of PAs was based on clinical manifestations, biochemical features of hormonal secretion, magnetic resonance imaging (MRI), and histopathological analyses confirmed by two pathologists after surgical resections. NIPA is defined as the limitation of tumor mass within the sellar region, without any compression on peripheral structures (Fig. 1A, B). The definition of IPA is according to Knosp classifications into grades III-IV [12] (Fig. 1C, D). IPAs ( $n=3)$ and NIPAs $(n=3)$ were selected for IncRNA microarrays. The details of these six PAs are shown in Table 1. Also, another 41 specimens of PAs, including IPAs $(n=21)$ and NIPAs $(n=16)$, were used for the validation by qRT-PCR. 
The clinical characteristics of the 41 patients with PAs are summarized in Table 2. Tumor dimensions were manually obtained from MRI. A microadenoma was defined by a maximal tumor diameter of $<10$ $\mathrm{mm}$, a macroadenoma was $\geq 10 \mathrm{~mm}$, a large macroadenoma was $\geq 20 \mathrm{~mm}$, while a giant adenoma was $\geq 40 \mathrm{~mm}$. The dimensional indices of the tumors were measured and recorded in three orthogonal planes, namely: transverse (TR), anteroposterior (AP), and craniocaudal (CC). The tumor volumes were estimated using the following formula: $V=(\pi \times[T R \times A P \times C C]) / 6$ [13].After surgical excision, all tissue samples were immediately frozen in liquid nitrogen and stored at $-80^{\circ} \mathrm{C}$ for further analyses. All procedures of this study were approved by the Ethics Committee of Guangdong Provincial People's Hospital. Informed consent was obtained from all patients.

\section{Total RNA extraction and purification}

Total RNA was extracted using Trizol reagent (Invitrogen, Carlsbad, USA) according to the manufacturer's protocol. Furthermore, the quantification of total RNA was evaluated by Bioanalyzer 2200 (Agilent, California, USA) and kept at $-80^{\circ} \mathrm{C}$. The RNA samples with RNA integrity number (RIN) $>6.0$ are acceptable for rRNA depletion and subsequent IncRNA purification. The purification of total RNA was validated by gel electrophoresis.

\section{cDNA library construction}

cDNA libraries were constructed for each pooled RNA sample using the NEBNext ${ }^{\circledR}$ Ultra ${ }^{\text {TM }}$ Directional RNA Library Prep Kit (New England BioLabs Inc., MA, US) for Illumina according to the manufacturer's instructions. The protocol comprises the following steps: mRNA molecules are fragmented into 150-200 bp using divalent cations at $94^{\circ} \mathrm{C}$ for $8 \mathrm{~min}$. The cleaved RNA fragments as templates were reversetranscribed into first-strand cDNA. Subsequently, the second-strand cDNA was synthesized using Polymerase I and RNase $\mathrm{H}$ with reaction buffer. Target bands were harvested through AMPure XP Beads (Beckman coulter). The products were then purified and enriched by PCR to create the final cDNA libraries and quantified by Agilent 2200. The tagged cDNA libraries were pooled in equal ratio and used for $150 \mathrm{bp}$ paired-end sequencing in a single lane of the Illumina HiSeq XTen. The experiments of library construction and RNA sequencing were completed at the Center of NovelBio Lab center of Novelbio lab (Shanghai, China).

\section{Mapping and identification of differentially expressed genes}

Before read mapping, clean reads were obtained from the raw reads by removing the adaptor sequences, reads with $>5 \%$ ambiguous bases (noted as $N$ ), and low-quality reads containing more than $20 \%$ of bases with qualities of $<20$. The clean reads were then aligned to the human genome (version: GRCh38 NCBI) using the HISAT2 [14]. HTSeq was used to count gene and IncRNA, while reads per kilobase per million mapped reads method was used to determine gene expression [15]. We applied DESeq algorithm [16] to filter the differentially expressed genes, after the analysis of the level of significance, i.e, determination of $P$ value, and false discovery rate (FDR) analysis under the following criteria: 1 ) fold change $>2$ or $<0.5,2)$ FDR $<0.05$ [17]. 


\section{Functional enrichment analysis}

Gene Ontology (GO) analysis was performed to facilitate elucidating the biological implications of unique genes [18]. We downloaded the GO annotations from NCBI (http://www.ncbi.nlm.nih.gov/), UniProt (http://www.uniprot.org/), and the Gene Ontology (http://www.geneontology.org/). Fisher's exact test was applied to identify the significant GO categories and FDR was used to correct the $P$ values. The Gene Ontology is structured as a directed acyclic graph, and each term has defined relationships to one or more other terms. GO-Tree was built based on the Gene Ontology Directed Acyclic Graph to provide userfriendly data navigation and visualization. We selected the significant GO-Term $(P$ value $<0.01)$ in GO analysis based on the differentially expressed genes to construct the GO-Tree for summarizing the function affected in the experiment [19].

Kyoto Encyclopedia of Genes and Genomes (KEGG) analyses were performed to clarify intuitively the role and significance of target genes in the overall biological pathways [20]. We selected the genes in enriched biological pathways and using Cytoscape for graphical representations of pathways [21]. The KEGG database was used to build the network of genes according to the relationship among the genes, proteins, and compounds in the database.

\section{Construction of the IncRNA-miRNA-target gene interaction network}

The role of IncRNAs in IPAs was investigated by a IncRNA-miRNA-target gene interaction network. According to the IncRNA microarray results, the 10 most dysregulated IncRNAs in IPAs were selected and Cytoscape software was performed to map out an interaction network. Putative interactions between IncRNAs and miRNAs were predicted using the online databases Jefferson Computational Medicine Center-RNA22 v2 microRNA target detection (https://cm.jefferson.edu/rna22/Interactive/) and LncBase Predicted v. 2 (http://carolina.imis.athena-innovation.gr/diana_tools/web/index.php? $r=$ Incbasev2\%2Findex-predicted). Then, the miRNA with the highest target scores were selected, and their target genes were evaluated by TargetScan [22] and miRanda [23]. Finally, miRNAs and their target genes with high targeting-relationship scores were selected to construct the IncRNA-miRNA-mRNA interaction network.The interaction network was delineated using the Cytoscape software.

\section{qRT-PCR assay for the validation}

Total RNA was extracted using Trizol reagent (Invitrogen) for 41 specimens of PAs. Reverse transcription and qRT-PCR were performed using a Reverse Transcription Kit (Takara, Dalian, China) and PrimeScript RT Reagent Kit (Takara, Dalian, China), respectively, as previously described [24]. The expression of IncRNA was measured by qRT-PCR. The sequences of the primers are listed in Table 3. The gene expression levels were normalized to actin. Gene expression levels were determined by the $2^{-\Delta \Delta \mathrm{Ct}}$ method and analyzed for statistical significance.

\section{Statistical analysis}


Measurement data are presented as mean \pm standard error of the mean (SEM) and enumeration data are presented as percentages. Comparisons were performed using independent sample t-test between pairs of groups or one-way analysis of variance for more than two groups followed by Dunnett's multiple comparison test. The receiver operating characteristic (ROC) curves and the area under the curve (AUC) were used to estimate the diagnostic power and accuracy of IncRNAs on invasive/noninvasive PAs. All statistical analyses were performed on Statistical Product and Service Solutions (SPSS) 25.0 software (SPSS Inc., Chicago, IL, USA). $P<0.05$ was considered statistically significant, which is indicated in the figures.

\section{Results}

\section{Identification of differentially expressed IncRNAs between IPAs and NIPAs}

To identify differentially expressed IncRNAs in IPAs and NIPAs, we performed a high-throughput human IncRNA microarray in three IPAs and three NIPAs. The correlation plot was used to detect the correlation between microarray samples and to confirm the homogeneity between biological replicates (Fig. $2 \mathrm{~A})$. The box plot demonstrated that the distributions of normalized intensities were almost identical among all samples (Fig. 2B). Furthermore, hierarchical clustering was used to illustrate significantly differential IncRNAs expression in the two groups (Fig. 2C), and the volcano plots were used to reveal the variations in IncRNAs expression levels between the two groups (Fig. 2D). Overall, 8872 IncRNAs and 16039 mRNAs were identified in PAs by human IncRNA microarray. Among these, 246 IncRNAs were differentially expressed in IPAs compared with NIPAs, including 81 upregulated IncRNAs and 165 downregulated ones (Fig. 2D). Meanwhile, 566 mRNAs were differentially expressed, including 289 upregulated mRNAs and 277 downregulated ones between the invasive and noninvasive tumor tissues. These results suggested that the expression of IncRNAs in IPAs was different from that matched NIPAs.

\section{Delineation of GO enrichment and KEGG pathway analysis}

To further investigate the functional roles of the differentially expressed IncRNAs, GO enrichment and KEGG pathway analyses were performed. GO terms are classified into three different domains, namely: biological processes (BP), molecular functions (MF), and cellular components (CC). The top 15 generally changed GO terms were ranked by fold enrichment or enrichment score as listed in Figure 3. The top five identified BP were post-translational protein modification, cellular protein metabolic process, regulation of inhibitory postsynaptic membrane, negative regulation of synapse assembly, and visual perception (Fig. 3A). The top five identified MF terms were RS domain binding, ligand-gated ion channel activity, cyclic guanosine monophosphate (cGMP) binding, uridine diphosphate (UDP) -glycosyltransferase activity, and ion channel binding (Fig. 3B). Furthermore, the top five identified CC terms were slit diaphragm, nuclear speck, dendrite, transmembrane transporter complex, and gamma-tubulin small complex (Fig. 3C).

The KEGG database was used to identify the pathways and molecular interactions associated with the target genes. Our data indicated that the target genes were mostly enriched in neuroactive ligand-receptor interaction, hypoxia-inducible factor 1alpha (HIF-1) signaling pathway, spliceosome, pathways in cancer, 
and $\mathrm{N}$-glycan biosynthesis (Fig. 4). According to these results, these pathways may contribute significantly to the invasiveness of PAs.

\section{Construction of the IncRNA-miRNA-mRNA coexpression network}

To reveal the potential functions and mechanisms of differently expressed IncRNAs in IPAs, a IncRNAmiRNA-mRNA coexpression network was subsequently constructed, based on bioinformatics analysis. The most down- and up-regulated IncRNAs in IPAs were selected for the IncRNA-miRNA-mRNA coexpression network, which is delineated using Cytoscape (Fig. 5).

\section{Validation of differentially expressed IncRNAs in PAs by qRT-PCR}

To validate the microarray results, three IncRNAs that exhibited significant changes in expression levels were selected for validation using qRT-PCR in 25 IPAs and 16 NIPAs, namely: FAM182B, LOC105371531, and LOC105375785. qRT-PCR results confirmed that all three IncRNAs were significantly $(P<$ 0.05) decreased in IPAs than in NIPAs (Fig. 6A-C). The results of qRT-PCR were consistent with the microarray expression data, confirming the high reliability of the microarray data.

\section{Diagnostic values of three selected IncRNAs for IPAs}

ROC curve analyses were performed to determine the diagnostic sensitivity and specificity of the three selected IncRNAs for IPAs (Fig. 6D-F). The AUCs for FAM182B, LOC105371531, and LOC105375785 were 0.798 (95\% confidence interval ( $95 \% \mathrm{Cl}$ ): 0.650-0.945), 0.730 (95\% Cl: $0.557-0.945)$, and 0.762 (95\% Cl: 0.604-0.921), respectively. These data suggested that FAM182B and LOC105375785 can be used to distinguish patients with IPAs from patients with NIPAs if AUC $\geq 0.75$ is considered diagnostically significant for the biomarker.

\section{Correlation between FAM182B expression and clinical features of patients with PAs}

In consideration of FAM182B with the highest value of AUC, we conducted further investigation on FAM182B. Using median values of FAM182B expression levels of all patients as the boundary line, we further divided the 41 patients with PAs into high-/low-expression subgroups of FAM182B. The analysis demonstrated that FAM182B expression had no association with gender, age, Ki67 percentage, and surgical extent, however, a significant relationship was found between invasive behavior and FAM182B ( $P$ $=0.001)$ (Table 4). The ratio of FAM182B high-expression in IPAs was significantly $(P=0.001)$ higher than that in patients with NIPA (Table 4).

\section{Discussion}

IncRNAs, a class of RNA transcripts longer than 200 nucleotides, can regulate the expression of proteincoding genes at the transcriptional and translational levels [25]. IncRNAs are involved in various processes related to gene regulation [26]. Accumulating evidence indicates that the expression of IncRNAs is associated with various tumors and can be a promising biomarker for the diagnosis of tumors [27]. Pituitary adenoma, one of the most common intracranial tumors, may invade the cranial bone, 
sphenoid bone, etc [3]. The mechanisms associated with the invasion of PAs and novel biomarkers for the diagnosis of IPAs remain largely unclear. Recently, several IncRNAs, including small nucleolar RNA host gene 1 (SNHG1), H19, colon cancer associated transcript 2 (CCAT2), LINC00473, and antisense noncoding RNA in the INK4 locus (ANRIL), participated in the proliferation, progression, and invasion of PAs [28-31]. However, the expression pattern of IncRNAs-mRNA and comprehensive analysis for dysregulated IncRNAs in IPAs remained to be investigated.

In this study, IncRNA microarray was performed to investigate the IncRNA expression pattern in the invasive and noninvasive PAs. We identified 246 IncRNAs that were differentially expressed at a significant level; 81 of which were upregulated and 165 were downregulated in IPAs. Then, these differentially expressed IncRNAs were integrated into hierarchical categories according to the heat maps. We observed that the IncRNA expression patterns were remarkably different between the invasive and noninvasive PAs. The results may indicate that IncRNAs are involved in regulating the invasiveness of PAs. These findings are consistent with previous reports, which reported that IncRNAs are involved in tumor invasive behaviors [32,33]. The results of the expression pattern of the differentially expressed IncRNAs and pathway analysis are different from the previous study analyzed using the GSE26966 database [9]. The basis of this difference may be because pituitary gonadotrope tumors were used for the microarray experiments in the GSE26966 database.

To further identify the potential function of these differentially expressed IncRNAs, GO enrichment and KEGG pathway analyses were performed. Notably, the most significant GO terms of IncRNAs were posttranslational protein modifications, which were previously reported to be important in the development of PAs [34]. KEGG pathway analysis for the differentially expressed IncRNAs revealed that neuroactive ligand-receptor interaction, HIF-1 signaling pathway may serve pivotal roles in the invasive mechanisms of PAs, as they were more likely to be identified in the IPAs than in NIPAs. Previous studies demonstrated that the expression levels of HIF-1 in IPAs were significantly higher than in NIPAs, and HIF-1 signaling pathway contributed to promoting the invasion of PAs [35, 36]. Hou et al. [37] identified that differentially expressed genes in pituitary gonadotroph adenomas were enriched in neuroactive ligand-receptor interaction pathway, which agrees with our results.

Further analysis of three dysregulated IncRNAs from the tissues of 25 IPAs and 16 NIPAs confirmed the reliability of the IncRNA microarray results. Additionally, the three validated IncRNAs, FAM182B, LOC105371531, and LOC105375785, were downregulated in IPAs and could distinguish IPAs from NIPAs. These results collectively demonstrated that IncRNAs may be implicated in the invasive behaviors of PAs. Accumulating evidence has also shown that IncRNAs can be promising biomarkers in various cancers $[38,39]$. For instance, Liu et al. [40] demonstrated that SNHG16 can be a potential biomarker in hepatocellular carcinoma, while Teng et al. [41] showed that lung cancer associated transcript 1 acts as a potential biomarker in gastric cancer. In our study, both FAM182B and LOC105375785 had relatively high specificity and sufficient sensitivity for the diagnosis of IPAs by ROC analysis. These results collectively demonstrated that IncRNAs may function as promising novel biomarkers for the diagnosis of IPAs. Moreover, FAM182B is associated with hepatocellular carcinoma [42]. In our study, FAM182B, with the 
highest value of AUC among the three validated IncRNAs, had a significant relationship with the invasive behavior of PAs.

Notably, there are two main limitations of this study. First, we did not perform the functional confirmation of these differentially expressed IncRNAs to clarify the functions and mechanisms of IncRNAs in the invasiveness of PAs. Second, the number of PAs is relatively small that may limit statistical power. The possible clinical implications of IncRNAs for the diagnosis of IPAs remain to be elucidated using a larger number of samples from patients with IPAs.

In conclusion, our results revealed the expression profile of differentially expressed IncRNAs in IPAs by microarray analysis. Furthermore, GO enrichment and KEGG pathway analyses were performed to identify the potential functions of differentially expressed IncRNAs. Additionally, coexpression networks were constructed for IncRNA-mRNA. Taken together, three validated IncRNAs, namely: FAM182B, LOC105371531, and LOC105375785 can be promising biomarkers in differentiating IPAs with NIPAs. Further investigations are needed to illustrate the detailed functions and mechanisms of IncRNAs in the invasive behaviors of PAs.

\section{Abbreviations}

IncRNAs

long non-coding RNAs

PAs

pituitary adenomas

NIPA

noninvasive pituitary adenoma

IPA

invasive pituitary adenoma

GO

Gene Ontology

KEGG

Kyoto Encyclopedia of Genes and Genomes

qRT-PCR

real-time quantitative reverse transcription polymerase chain reaction

ROC

receiver operating characteristic

AUC

area under the curve

MRI

magnetic resonance imaging

TR

transverse 
AP

anteroposterior

CC

craniocaudal

RIN

RNA integrity number

FDR

false discovery rate

SPSS

Statistical Product and Service Solutions

BP

biological processes

$\mathrm{MF}$

molecular functions

CC

cellular components

\section{Declarations}

\section{Ethics approval and consent to participate}

All procedures of this study were approved by the Ethics Committee of Guangdong Provincial People's Hospital. Informed consent was obtained from all patients.

All methods were performed in accordance with the relevant guidelines and regulations.

\section{Consent for publication}

Not applicable.

\section{Competing interests}

The authors declare that they have no competing interests.

\section{Funding}

This work was supported by National Natural Science Foundation of China (grants number: 81800682 to Yanli Li); the Medical and Health Project of Guangzhou (grants number: 20201A011079 to Yanli Li); the Medical Science and Technology Research Fund Project of Guangdong (grants number: A2018443 to Yanli Li). 


\section{Author contributions}

Chao Peng and Shuaikai Wang performed the experiments, prepared figures and tables, and wrote the manuscript. Jinxiu Yu analyzed the data. Xiaoyi Deng, Zhishan Chen, Huiyu Ye, Hongru Yao and Hanjia Cai collected the data and performed the experiments. Yong Yuan and Yanli Li conceived and designed the study. All the authors agreed on the final manuscript.

\section{Acknowledgements}

Not applicable.

\section{Data availability statement}

The datasets used and analyzed during the current study are available on the GEO database under the accession number GSE 191113 (https://www.ncbi.nlm.nih.gov/geo/query/acc.cgi?acc=GSE191113).

\section{References}

1. Ezzat S, Asa SL, Couldwell WT, Barr CE, Dodge WE, Vance ML, McCutcheon IE: The prevalence of pituitary adenomas: a systematic review. Cancer 2004, 101:613-619.

2. Lopes MBS: World Health Ozganization 2017 Classification of Pituitary Tumors. Endocrinol Metab Clin North Am 2020, 49:375-386.

3. Miermeister CP, Petersenn S, Buchfelder M, Fahlbusch R, Lüdecke DK, Hölsken A, Bergmann M, Knappe UJ, Hans VH, Flitsch J, et al: Erratum: Histological criteria for atypical pituitary adenomas-data from the German pituitary adenoma registry suggests modifications. Acta Neuropathol Commun 2016, 4:21.

4. Ponting CP, Oliver PL, Reik W: Evolution and functions of long noncoding RNAs. Cel/ 2009, 136:629641.

5. Peng WX, Koirala P, Mo YY: LncRNA-mediated regulation of cell signaling in cancer. Oncogene 2017, 36:5661-5667.

6. Li Y, Lu L, Wu X, Li Q, Zhao Y, Du F, Chen Y, Shen J, Xiao Z, Wu Z, et al: The Multifaceted Role of Long Non-Coding RNA in Gastric Cancer: Current Status and Future Perspectives. Int J Biol Sci 2021, 17:2737-2755.

7. Nong S, Chen X, Wang Z, Xu G, Wei W, Peng B, Zhou L, Wei L, Zhao J, Wei Q, et al: Potential IncRNA Biomarkers for HBV-Related Hepatocellular Carcinoma Diagnosis Revealed by Analysis on Coexpression Network. Biomed Res Int 2021, 2021:9972011.

8. Li L, Wei H, Zhang YW, Zhao S, Che G, Wang Y, Chen L: Differential expression of long non-coding RNAs as diagnostic markers for lung cancer and other malignant tumors. Aging (Albany NY) 2021, 13. 
9. Xue YH, Ge YQ: Construction of IncRNA regulatory networks reveal the key IncRNAs associated with Pituitary adenomas progression. Math Biosci Eng 2020, 17:2138-2149.

10. Guo J, Fang Q, Liu Y, Xie W, Zhang Y, Li C: Identifying critical protein-coding genes and long noncoding RNAs in non-functioning pituitary adenoma recurrence. Oncol Lett 2021, 21:264.

11. Zhu HB, Li B, Guo J, Miao YZ, Shen YT, Zhang YZ, Zhao P, Li CZ: LncRNA MEG8 promotes TNF-a expression by sponging miR-454-3p in bone-invasive pituitary adenomas. Aging (Albany NY) 2021, 13:14342-14354.

12. Micko AS, Wöhrer A, Wolfsberger S, Knosp E: Invasion of the cavernous sinus space in pituitary adenomas: endoscopic verification and its correlation with an MRI-based classification. $J$ Neurosurg 2015, 122:803-811.

13. Mak HK, Lai SW, Qian W, Xu S, Tong E, Vance ML, Oldfield E, Jane J, Jr., Sheehan J, Yau KK, Wintermark M: Effective time window in reducing pituitary adenoma size by gamma knife radiosurgery. Pituitary 2015, 18:509-517.

14. Trapnell C, Roberts A, Goff L, Pertea G, Kim D, Kelley DR, Pimentel H, Salzberg SL, Rinn JL, Pachter L: Differential gene and transcript expression analysis of RNA-seq experiments with TopHat and Cufflinks. Nat Protoc 2012, 7:562-578.

15. Anders S, PyI PT, Huber W: HTSeq-a Python framework to work with high-throughput sequencing data. Bioinformatics 2015, 31:166-169.

16. Anders S, Huber W: Differential expression analysis for sequence count data. Genome Bio/2010, 11:R106.

17. Benjamini Y, Drai D, Elmer G, Kafkafi N, Golani I: Controlling the false discovery rate in behavior genetics research. Behav Brain Res 2001, 125:279-284.

18. Ashburner M, Ball CA, Blake JA, Botstein D, Butler H, Cherry JM, Davis AP, Dolinski K, Dwight SS, Eppig JT, et al: Gene ontology: tool for the unification of biology. The Gene Ontology Consortium. Nat Genet 2000, 25:25-29.

19. Zhang B, Schmoyer D, Kirov S, Snoddy J: GOTree Machine (GOTM): a web-based platform for interpreting sets of interesting genes using Gene Ontology hierarchies. BMC Bioinformatics 2004, 5:16.

20. Kanehisa M, Goto S: KEGG: kyoto encyclopedia of genes and genomes. Nucleic Acids Res 2000, 28:27-30.

21. Shannon P, Markiel A, Ozier O, Baliga NS, Wang JT, Ramage D, Amin N, Schwikowski B, Ideker T: Cytoscape: a software environment for integrated models of biomolecular interaction networks. Genome Res 2003, 13:2498-2504.

22. Enright AJ, John B, Gaul U, Tuschl T, Sander C, Marks DS: MicroRNA targets in Drosophila. Genome Biol 2003, 5:R1.

23. Pasquinelli AE: MicroRNAs and their targets: recognition, regulation and an emerging reciprocal relationship. Nat Rev Genet 2012, 13:271-282. 
24. Su Y, Jiang X, Li Y, Li F, Cheng Y, Peng Y, Song D, Hong J, Ning G, Cao Y, Wang W: Maternal Low Protein Isocaloric Diet Suppresses Pancreatic $\beta$-Cell Proliferation in Mouse Offspring via miR-15b. Endocrinology 2016, 157:4782-4793.

25. Chen LL: Linking Long Noncoding RNA Localization and Function. Trends Biochem Sci 2016, 41:761772.

26. Clark MB, Mattick JS: Long noncoding RNAs in cell biology. Semin Cell Dev Bio/ 2011, 22:366-376.

27. Liu B, Xiang W, Liu J, Tang J, Wang J, Liu B, Long Z, Wang L, Yin G, Liu J: The regulatory role of antisense IncRNAs in cancer. Cancer Cell Int 2021, 21:459.

28. Wang H, Wang G, Gao Y, Zhao C, Li X, Zhang F, Jiang C, Wu B: Lnc-SNHG1 Activates the TGFBR2/SMAD3 and RAB11A/Wnt/ $\beta$-Catenin Pathway by Sponging MiR-302/372/373/520 in Invasive Pituitary Tumors. Cell Physiol Biochem 2018, 48:1291-1303.

29. Wu ZR, Yan L, Liu YT, Cao L, Guo YH, Zhang Y, Yao H, Cai L, Shang HB, Rui WW, et al: Inhibition of mTORC1 by IncRNA H19 via disrupting 4E-BP1/Raptor interaction in pituitary tumours. Nat Commun 2018, 9:4624.

30. Fu D, Zhang Y, Cui H: Long noncoding RNA CCAT2 is activated by E2F1 and exerts oncogenic properties by interacting with PTTG1 in pituitary adenomas. Am J Cancer Res 2018, 8:245-255.

31. Li J, Qian Y, Zhang C, Wang W, Qiao Y, Song H, Li L, Guo J, Lu D, Deng X: LncRNA LINC00473 is involved in the progression of invasive pituitary adenoma by upregulating KMT5A via ceRNAmediated miR-502-3p evasion. Cell Death Dis 2021, 12:580.

32. Ouyang J, Zhong Y, Zhang Y, Yang L, Wu P, Hou X, Xiong F, Li X, Zhang S, Gong Z, et al: Long noncoding RNAs are involved in alternative splicing and promote cancer progression. Br J Cancer 2021.

33. Yadav G, Kulshreshtha R: Metastasis associated long noncoding RNAs in glioblastoma: Biomarkers and therapeutic targets. J Cell Physiol 2021.

34. Li J, Zhan X: Mass spectrometry-based proteomics analyses of post-translational modifications and proteoforms in human pituitary adenomas. Biochim Biophys Acta Proteins Proteom 2021, 1869:140584.

35. He W, Huang L, Shen X, Yang Y, Wang D, Yang Y, Zhu X: Relationship between RSUME and HIF1a/VEGF-A with invasion of pituitary adenoma. Gene 2017, 603:54-60.

36. Xiao Z, Liu Q, Zhao B, Wu J, Lei T: Hypoxia induces hemorrhagic transformation in pituitary adenomas via the HIF-1a signaling pathway. Oncol Rep 2011, 26:1457-1464.

37. Hou Z, Yang J, Wang G, Wang C, Zhang H: Bioinformatic analysis of gene expression profiles of pituitary gonadotroph adenomas. Oncol Lett 2018, 15:1655-1663.

38. Lu C, Wei D, Zhang Y, Wang P, Zhang W: Long Non-Coding RNAs as Potential Diagnostic and Prognostic Biomarkers in Breast Cancer: Progress and Prospects. Front Onco/ 2021, 11:710538.

39. Aurilia C, Donati S, Palmini G, Miglietta F, Falsetti I, lantomasi T, Brandi ML: Are Non-Coding RNAs Useful Biomarkers in Parathyroid Tumorigenesis? Int J Mol Sci 2021, 22. 
40. Liu Q, Gao P, Li Q, Xu C, Qu K, Zhang J: Long non-coding RNA SNHG16 as a potential biomarker in hepatocellular carcinoma: A meta-analysis. Medicine (Baltimore) 2021, 100:e27178.

41. Teng YY, Yu HJ, Ni LY, Lu GR, Zhu YS, Zhou LL, Zhang J, Xue ZX, Cai ZZ, Xing C: IncRNA LUCAT1 acts as a Potential Biomarker and Demonstrates Malignant Biological Behaviors in Gastric Cancer. Clin Lab 2021, 67.

42. Liu J, Li W, Zhang J, Ma Z, Wu X, Tang L: Identification of key genes and long non-coding RNA associated ceRNA networks in hepatocellular carcinoma. PeerJ 2019, 7:e8021.

\section{Tables}

Table 1. Details of three IPAs and three NIPAs used for microarray analysis.

\begin{tabular}{llllll} 
ID & Sex & $\begin{array}{l}\text { Age } \\
(\text { years })\end{array}$ & $\begin{array}{l}\text { Tumor size } \\
(\mathrm{mm})\end{array}$ & $\begin{array}{l}\text { Secretory function } \\
\text { Knosp } \\
\text { grade }\end{array}$ \\
\hline IPA-1 & male & 21 & $25 * 20 \star 38$ & Nonfunctioning & IV \\
\hline IPA-2 & male & 50 & $24 \star 26 * 18$ & Nonfunctioning & III \\
\hline IPA-3 & female & 57 & $23 \star 22 * 18$ & Nonfunctioning & IV \\
\hline NIPA-1 & male & 41 & $35 \star 24 \star 25$ & Nonfunctioning & I \\
\hline NIPA-2 & male & 56 & $29 \star 26 * 19$ & Nonfunctioning & I \\
\hline NIPA-3 & female & 38 & $14 * 18 * 18$ & Nonfunctioning & II
\end{tabular}

Table 2. Characteristics of 41 patients with PAs. 


\begin{tabular}{|c|c|c|}
\hline Characteristic & NIPA & IPA \\
\hline$N$ & 16 & 25 \\
\hline Age (years) & $43.56 \pm 10.46$ & $46.08 \pm 14.04$ \\
\hline Male, no (\%) & $11(68.8)$ & $14(56.0)$ \\
\hline \multicolumn{3}{|l|}{ Secretory function, n (\%) } \\
\hline Nonfunctioning & $9(56.2)$ & $16(64.0)$ \\
\hline PRL & $1(6.3)$ & $5(20.0)$ \\
\hline $\mathrm{GH}$ & $4(25.0)$ & $2(8.0)$ \\
\hline ACTH & $2(12.5)$ & $1(4.0)$ \\
\hline TSH & 0 & $1(4.0)$ \\
\hline Median tumor volume $\left(\mathrm{cm}^{3}\right)$ & $4.60(0.18-22.05)$ & $4.61(1.41-11.08)$ \\
\hline \multicolumn{3}{|l|}{ Tumor size, n (\%) } \\
\hline Microadenoma & 0 & 0 \\
\hline Macroadenoma & $5(31.3)$ & $1(4.0)$ \\
\hline Large macroadenoma & $9(56.2)$ & $20(80.0)$ \\
\hline Giant adenoma & $2(12.5)$ & $4(16.0)$ \\
\hline \multicolumn{3}{|l|}{ Surgical extent, no (\%) } \\
\hline Gross total resection & $14(87.5)$ & $17(68.0)$ \\
\hline Residual & $2(12.5)$ & $8(32.0)$ \\
\hline
\end{tabular}

PRL, prolactin hormone; GH, growth hormone; $\mathrm{ACTH}$, adrenocorticotropic hormone; TSH, thyroidstimulating hormone.

Table 3. Oligonucleotide sequences of primer for qRT-PCR analysis.

\begin{tabular}{lll} 
Gene symbol & Forward primer & Reverse primer \\
\hline Actin & TGTGGATCGGTGGCTCCATCCT & AAACGCAGCTCAGTAACAGTCCGC \\
\hline FAM182B & GCACTCTGGGTCCTGTTCTC & CACTTCCCTGCCTCCTACAC \\
\hline LOC105371531 & CAGGGTTATGAGATCGTC & GTTTCTGGGTCTTGGAGT \\
\hline LOC105375785 & ATCATCACTCTGCCCACCAT & AGTCGGATGACCTCCTCCTT
\end{tabular}


Table 4. Association between FAM182B expression and clinical characteristics

\begin{tabular}{|c|c|c|c|c|}
\hline \multirow[t]{3}{*}{ Variable } & \multicolumn{2}{|c|}{ FAM182B expression } & \multicolumn{2}{|c|}{ Univariate analysis } \\
\hline & Low & High & $c^{2}$ & $P$ value \\
\hline & $(n=21)$ & $(n=20)$ & & \\
\hline \multicolumn{5}{|l|}{ Gender } \\
\hline Male & $14(66.7 \%)$ & $11(55.0 \%)$ & \multirow[t]{2}{*}{0.586} & \multirow[t]{2}{*}{0.444} \\
\hline female & $7(33.3 \%)$ & $9(45.0 \%)$ & & \\
\hline \multicolumn{5}{|l|}{ Age (years) } \\
\hline$\nabla 44$ & $8(38.1 \%)$ & $12(60.0 \%)$ & \multirow[t]{2}{*}{1.967} & \multirow[t]{2}{*}{0.161} \\
\hline$\geq 44$ & $13(61.9 \%)$ & $8(40.0 \%)$ & & \\
\hline \multicolumn{5}{|l|}{ Ki67 (\%) } \\
\hline$\otimes 3$ & $15(71.4 \%)$ & $15(75.0 \%)$ & \multirow[t]{2}{*}{0.067} & \multirow[t]{2}{*}{0.796} \\
\hline$\geq 3$ & $6(28.6 \%)$ & $5(25.0 \%)$ & & \\
\hline \multicolumn{5}{|c|}{ Surgical extent } \\
\hline Residual & $7(33.3 \%)$ & $3(15.0 \%)$ & 2.012 & 0.156 \\
\hline Gross total & $14(66.7 \%)$ & $17(85.0 \%)$ & & \\
\hline \multicolumn{5}{|c|}{ Invasiveness } \\
\hline No & $3(14.3 \%)$ & $13(65.0 \%)$ & 11.072 & 0.001 \\
\hline Yes & $18(85.7 \%)$ & $7(35.0 \%)$ & & \\
\hline
\end{tabular}

Figures 
Figure 1
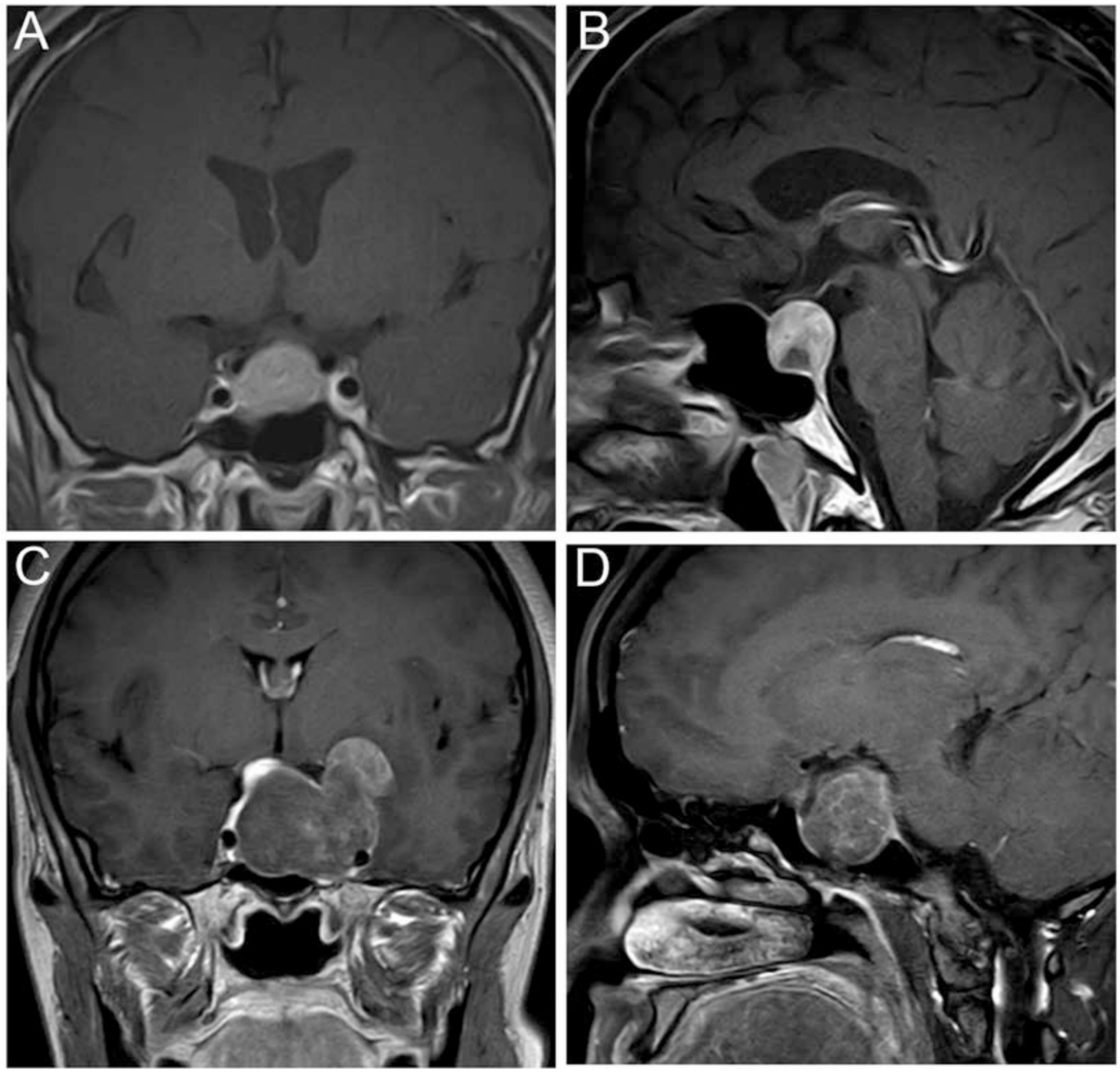

\section{Figure 1}

Enhanced magnetic resonance imaging of two patients with noninvasive pituitary adenoma (NIPA) and invasive pituitary adenoma (IPA), respectively. (A, B) Coronal and sagittal scans of NIPAs, respectively. (C, D) Coronal and sagittal scans of IPAs invaded the left cavernous sinus to surround the internal carotid artery (Knosp classification grades IV). 
Figure 2

A

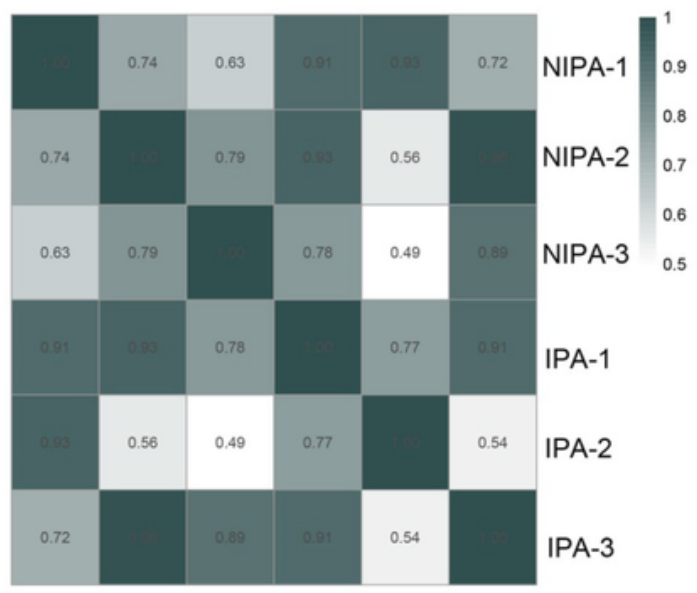

NIPA-1 NIPA-2 NIPA-3 IPA-1 IPA-2 IPA-3
B RPKM distribution

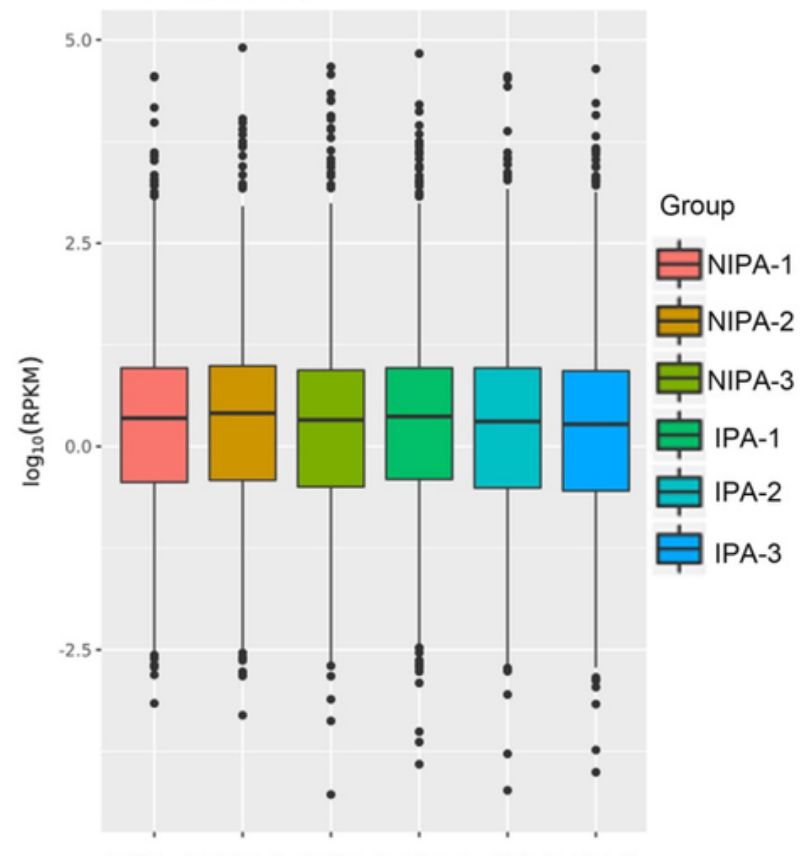

NIPA-1 NIPA-2 NIPA-3 IPA-1 IPA-2 IPA-3
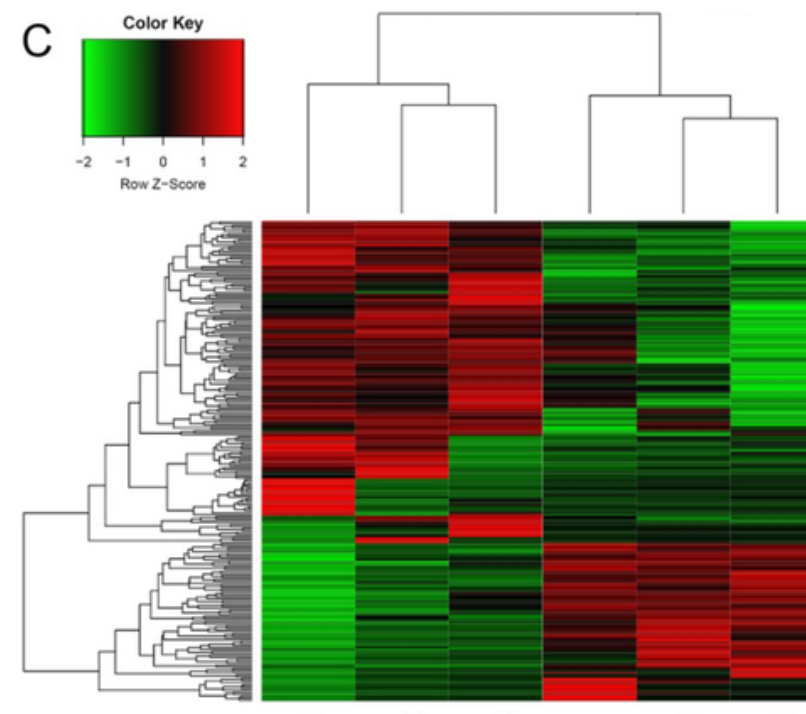

$\mathrm{D}$

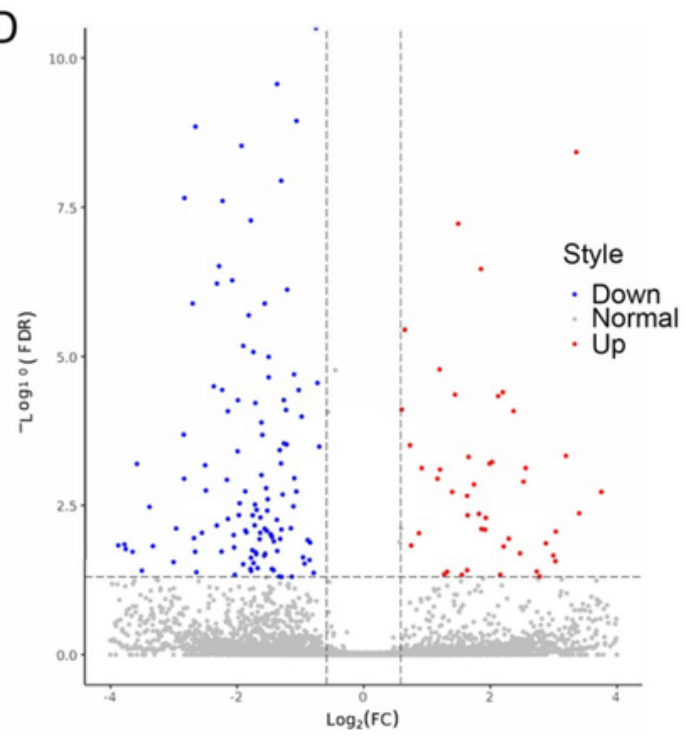

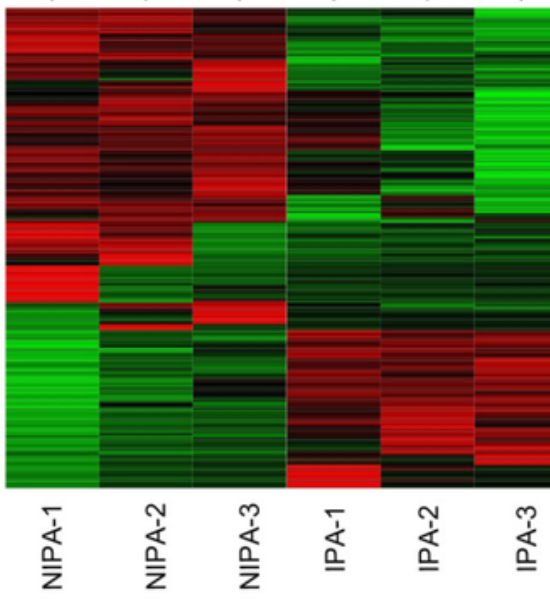

\section{Figure 2}

Long non-coding RNAs (IncRNA) profiles based on microarray. (A) The correlation among the six samples based on expression values of differentially expressed IncRNAs. (B) The boxplots of microarray intensity values. (C) Heat map based on the expression values of significantly altered IncRNAs with fold changes > 2 or $<0.5$ and $P<0.05$. Red and green indicate increased and decreased expression levels, respectively.

(D) Volcano plot showing differently expressed IncRNAs between invasive pituitary adenomas and 
noninvasive pituitary adenomas, with red dots indicating upregulation and green dots indicating downregulation.

\section{Figure 3}
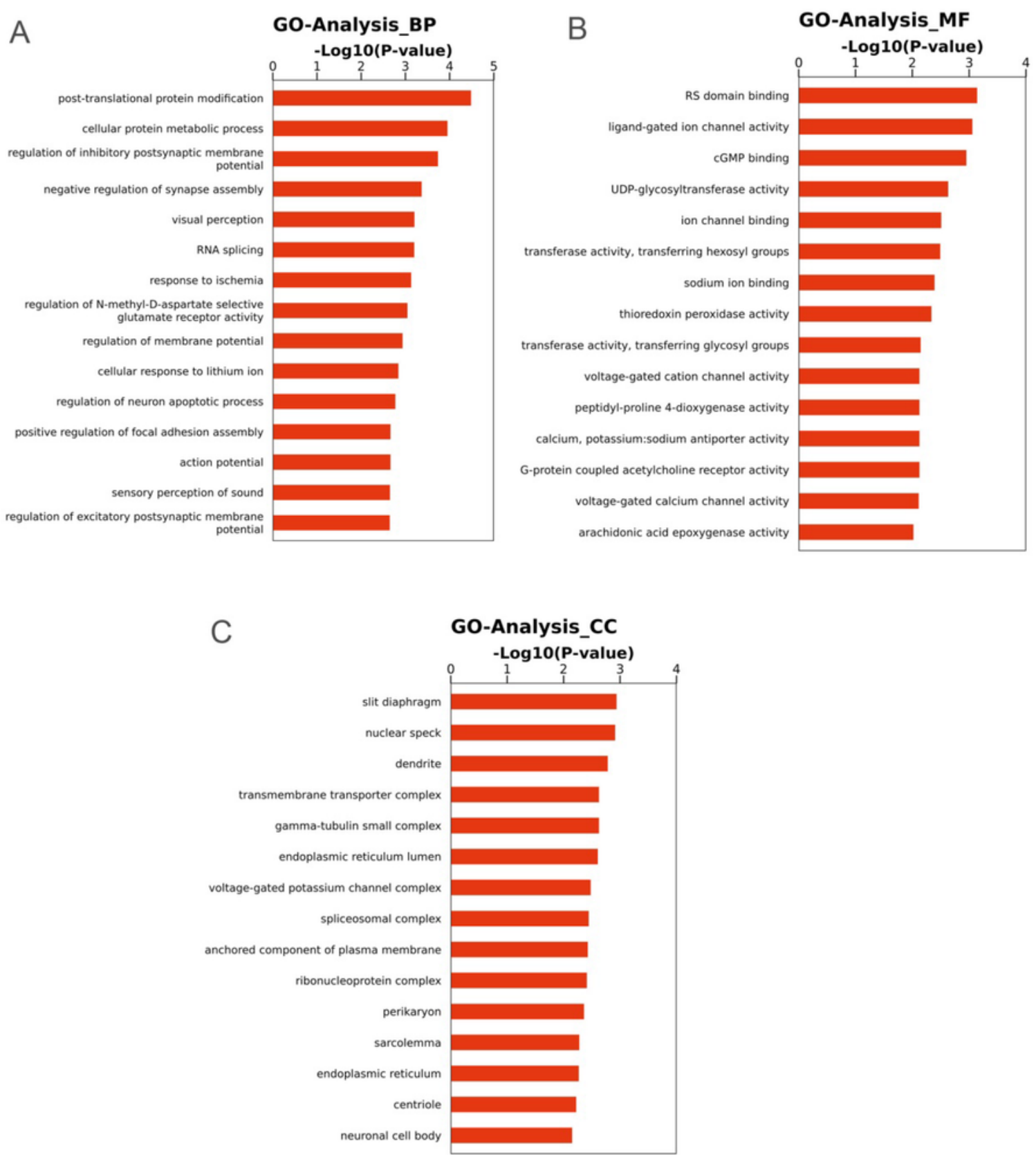

\section{Figure 3}

Gene ontology (GO) terms from BP (A), MF (B), and CC (C). Functional analysis of differentially expressed IncRNAs and co-expressed mRNAs for GO enrichment. BP, biological processes, MF, molecular functions, 
$\mathrm{CC}$, cellular components.

\section{Figure 4}

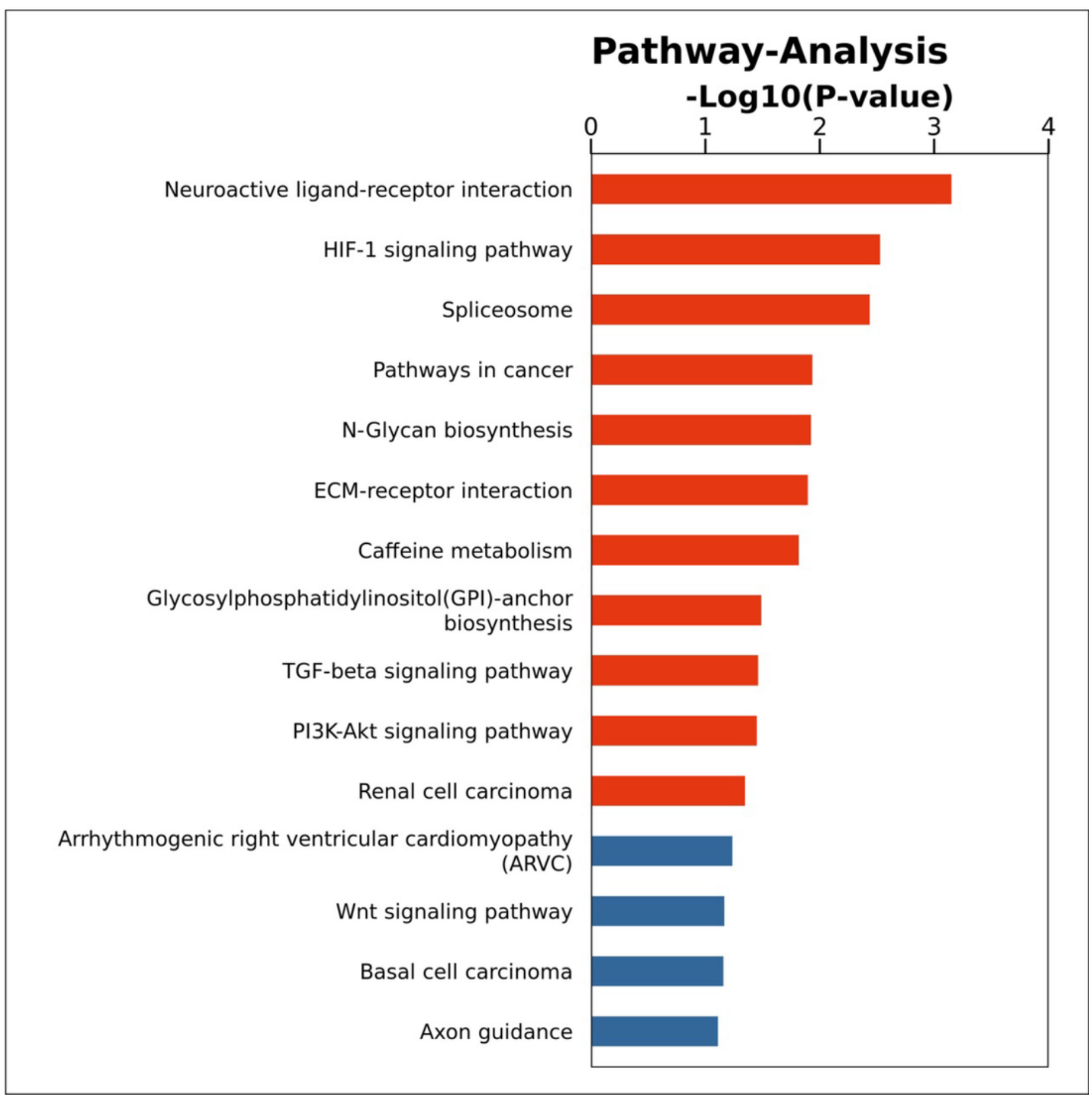

Figure 4

Kyoto Encyclopedia of Genes and Genomes (KEGG) pathway analysis of differentially expressed long non-coding RNAs (IncRNAs) and co-expressed mRNAs. The length of the column indicates the $P$ value. The longer the column and the lower the $P$ value, the more enriched and meaningful the pathway. 
Figure 5

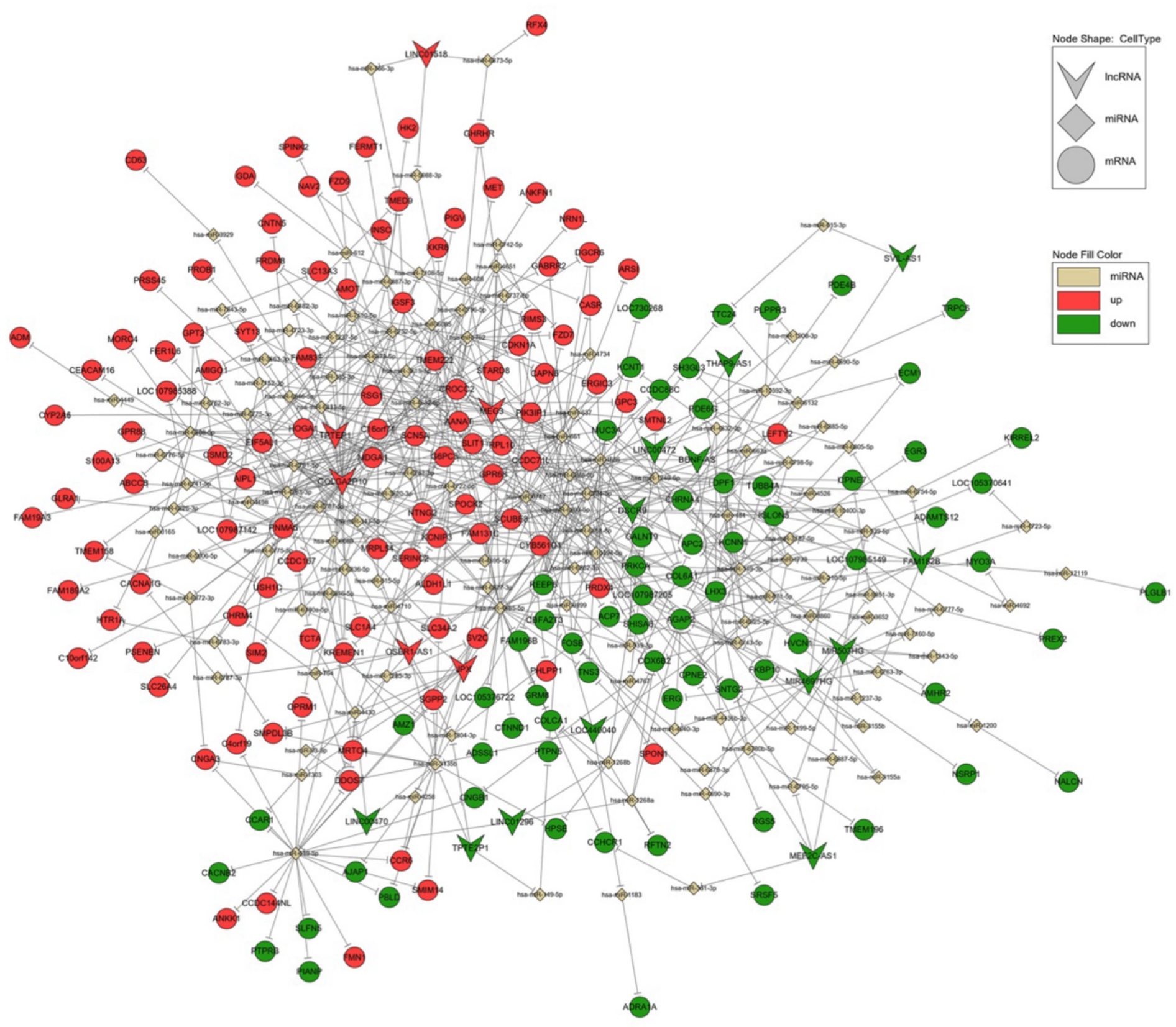

Figure 5

The interaction network of long non-coding RNAs (IncRNAs)-miRNA-target genes. The arrows, rhombus, and circle indicate IncRNA, miRNA, and mRNA, respectively. Red and blue colors designate upregulation and downregulation, respectively. 
Figure 6
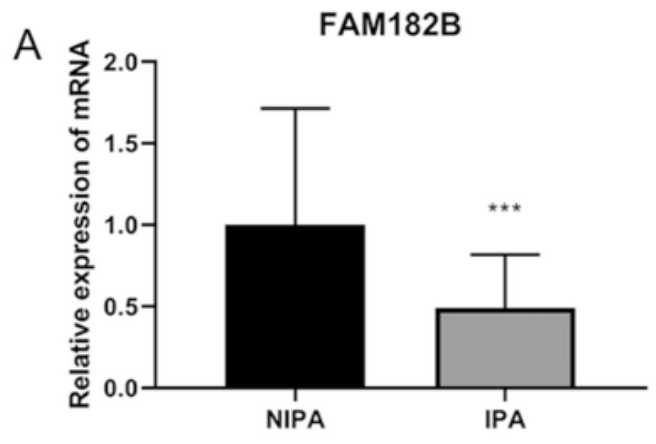

C
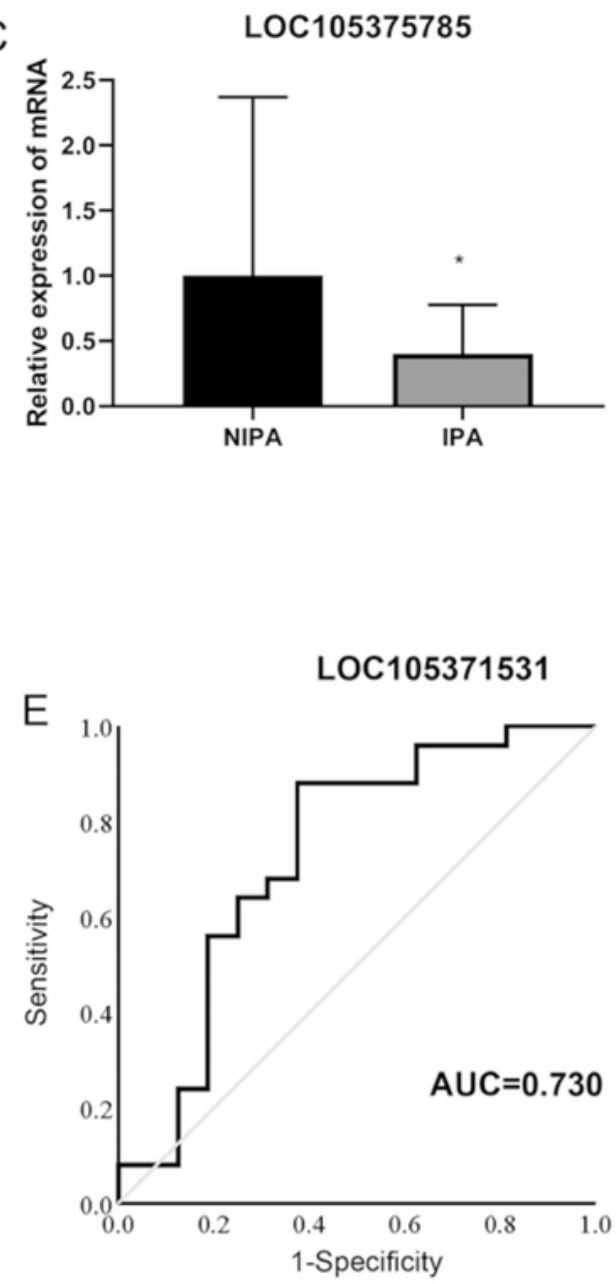

B

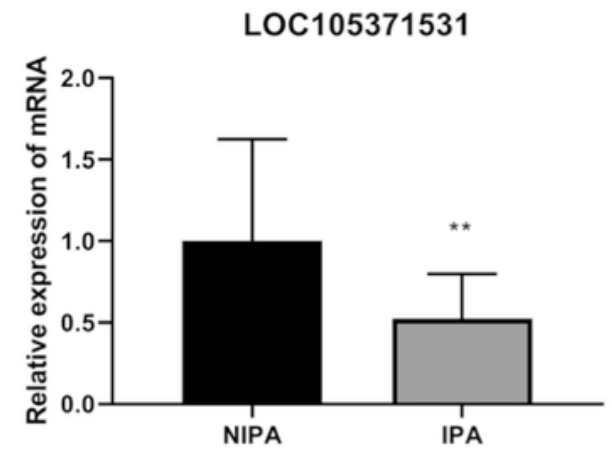

FAM182B

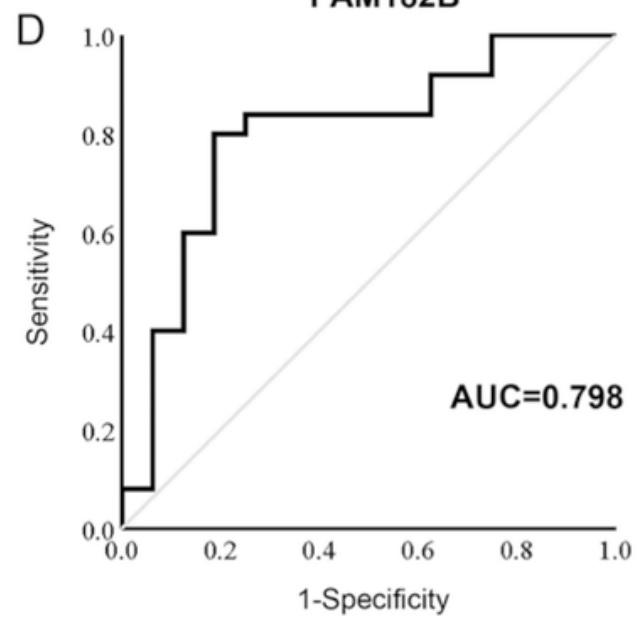

LOC105375785

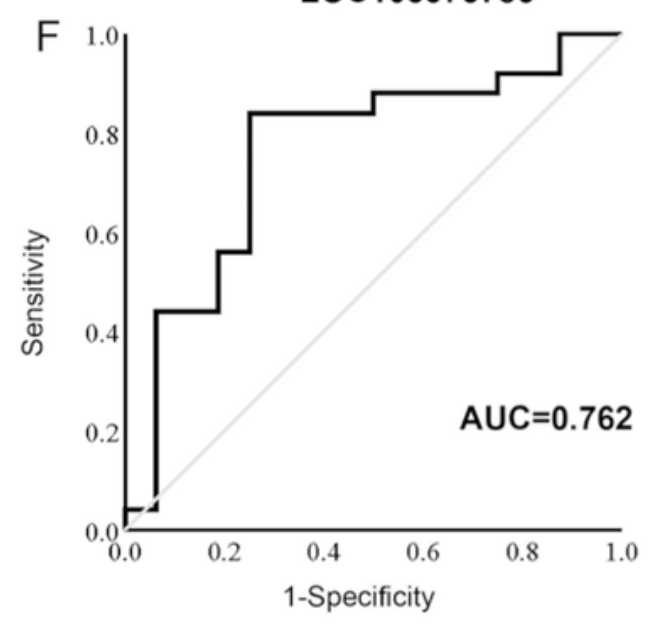

\section{Figure 6}

Validation of long non-coding RNAs (IncRNAs) microarray results by real-time quantitative reverse transcription polymerase chain reaction (qRT-PCR) and receiver operating characteristic (ROC) analysis for the three selected IncRNAs. (A-C) The expression levels of the three selected IncRNAs, including FAM182B, LOC105371531, and LOC105375785 in invasive pituitary adenoma (IPAs) ( $n=25)$ and noninvasive pituitary adenoma (NIPAs) $(n=16)$. (D-F) ROC curves of the selected three IncRNAs between 
IPAs and NIPAs. Data is represented as mean \pm SEM, $* P<0.05$, ${ }^{\star} P<0.01$, and $\star \star \star * P<0.001$ versus the NIPAs group. 